\title{
Ventricle wall dissection and vascular preservation with the pulsed water jet device: novel tissue dissector for flexible neuroendoscopic surgery
}

\author{
Tomohiro Kawaguchi, MD, PhD, Atsuhiro Nakagawa, MD, PhD, Toshiki Endo, MD, PhD, \\ Miki Fujimura, MD, PhD, Yukihiko Sonoda, MD, PhD, and Teiji Tominaga, MD, PhD \\ Department of Neurosurgery, Tohoku University Graduate School of Medicine, Sendai, Miyagi, Japan
}

\begin{abstract}
OBJECTIVE Neuroendoscopic surgery allows minimally invasive surgery, but lacks effective methods to control bleeding. Water jet dissection with continuous flow has been used in liver and kidney surgery since the 1980s, and is effective for tissue manipulation with vascular preservation, but involves some potential risks, such as elevation of intracranial pressure during application in the ventricles. The authors previously reported the efficacy of the actuator-driven pulsed water jet device (ADPJ) to dissect soft tissue with vascular preservation in microscopic neurosurgery. This feasibility study investigated the use of the ADPJ to reduce the amount of water usage, leading to more safety with sustained efficacy.

METHODS A small-diameter pulsed water jet device was developed for use with the flexible neuroendoscope. To identify the optimal conditions for the water jet, the flow rate, water pressure, and distance between the nozzle and target were analyzed in an in vitro study by using a gelatin brain phantom. A ventricle model was used to monitor the internal pressure and temperature. For ex vivo experiments the porcine brain was harvested and ventricle walls were exposed, and subsequently immersed into physiological saline. For in vivo experiments the cortex was microsurgically resected to make the small cortico-ventricle window, and then the endoscope was introduced to dissect ventricle walls.
\end{abstract}

RESULTS In the in vitro experiments, water pressure was approximately 6.5 bar at $0.5 \mathrm{~mm}$ from the ADPJ nozzle and was maintained at $1 \mathrm{~mm}$, but dropped rapidly toward $50 \%$ at $2 \mathrm{~mm}$, and became $10 \%$ at $3.5 \mathrm{~mm}$. The ADPJ required less water to achieve the same dissection depth compared with the continuous-flow water jet. With the ventricle model, the internal pressure and temperature were well controlled at the baseline, with open water drainage. These results indicated that the ADPJ can be safely applied within the ventricles. The ADPJ was introduced into a flexible endoscope and the ventricle walls were dissected in both the ex vivo and in vivo conditions. The ventricle wall was dissected without obscuring the view, and the vascular structures were anatomically preserved under direct application. Histological examination revealed that both the vessels on the ventricle wall and the fine vessels in the parenchyma were preserved.

CONCLUSIONS The ADPJ can safely and effectively dissect the ventricle wall, with vascular preservation in immersed conditions. To achieve the optimal result of tissue dissection with minimal surgical risk, the ADPJ is a potential device for neuroendoscopic surgery of the ventricles.

http://thejns.org/doi/abs/10.3171/2015.3.JNS142121

KEY WORDS pulsed water jet; neuroendoscope; minimally invasive surgery; ventricle tumor; pig; diagnostic and operative techniques

$\mathrm{M}$ INIMALLY invasive surgery is the goal of many recent advances in surgical devices. Neuroendoscopic surgery is now widely indicated for the surgical treatment of various diseases. Intraventricular lesions are often indicated for the neuroendoscopic approach, but tissue dissection performed using the flexible endoscope in the ventricles is still challenging because the number of instruments that can be used through the work- ing channel is restricted. Moreover, the lack of effective devices to control bleeding remains a problem with neuroendoscopic surgery, so that strategies to avoid bleeding are extremely important.

Water jet dissection has been used in liver and kidney surgery since the $1980 \mathrm{~s},{ }^{4,5,12-14}$ This technique initially used high-pressure continuous flow and could dissect tissue but preserve the vasculature, resulting in reductions in blood

ABBREVIATIONS ADPJ = actuator-driven pulsed water jet device; ICP = intracranial pressure.

SUBMITTED September 10, 2014. ACCEPTED March 30, 2015.

INCLUDE WHEN CITING Published online September 25, 2015; DOI: 10.3171/2015.3.JNS142121. 
loss, operative time, and surgical complications., ${ }^{9,12,19,20}$ However, the continuous-flow water jet device has several problems to be resolved, because a high volume of water supply is required for dissection, and the consequent water vortex tends to disperse tissue debris. ${ }^{1,3}$ Although the possible usefulness of a water jet dissector with a neuroendoscope has been reported previously, ${ }^{6}$ these characteristics can risk elevation of intracranial pressure (ICP) and obscuring of the neuroendoscopic view during ventricle wall dissection.

Our group has developed the actuator-driven pulsed water jet device (ADPJ) to dissect soft tissue..$^{18}$ Initially, the ADPJ was applied in glioma and pituitary surgery, which achieved fine vessel preservation and increased resection rate. ${ }^{8,10,11}$ The ADPJ is useful for microscopic neurosurgery and submucosal dissection of the esophagus, $, 7,8,15$ but no water jet device has been used in the ventricles with the flexible endoscope. Therefore, whether ADPJ can effectively dissect tissues immersed in fluids, and is safe for dissection of intraventricular lesions, remains unknown.

The present feasibility study investigated whether the ADPJ can maintain the efficacy of dissection properties and reduce the amount of water required when using the flexible neuroendoscope.

\section{Methods}

\section{Use of ADPJ for Neuroendoscopy}

A small-diameter pulsed water jet device was developed for use with the flexible neuroendoscope. The nozzle on the tip was $0.15 \mathrm{~mm}$ in inner diameter and $0.75 \mathrm{~mm}$ long. The wired connecting pipe was made of polyetheretherketone resin. The piezoelectric actuator unit was attached on the other end of the connecting pipe to drive the water jet. The input voltage of the actuator can range from 0 to $150 \mathrm{~V}$, and in the present study we basically used the following settings: voltage $30 \mathrm{~V}$, flow rate $5 \mathrm{ml} / \mathrm{min}$, and frequency $200 \mathrm{~Hz} .{ }^{18}$ This actuator can also provide a continuous-flow water jet. Details of the device are described elsewhere ${ }^{18}$ (Fig. 1).

\section{Mechanical Profile of ADPJ}

The ADPJ was used to eject $0.016 \mathrm{wt} \%$ methylene blue (methylene blue weight/total weight) into the test chamber filled with physiological saline. The high-speed camera was operated at a frame rate of 63,000 frames/sec and an interframe time of $15.9 \mu \mathrm{sec}$, with image resolution of 32 $\times 512$ pixels. The distance between the nozzle and target (standoff distance) ranged from 0.5 to $4.5 \mathrm{~mm}$. A pressure sensor (Quartz High-Pressure Sensor, Type 601A; Kistler) was used to measure the deflection pressure. The distance and the water pressure at each level were plotted.

\section{Evaluation of Dissection Profile in Brain Phantom Experiments}

Experimental brain phantoms were made with gelatin. Nitta gelatin 1st Grade (G1211; Nitta Gelatin, Inc.), 3.5 $\mathrm{wt} \%$ (gelatin weight/total weight) was dissolved in warm water and cooled to solidify at $280 \mathrm{~K}$. Gelatin blocks with this composition have equivalent mechanical profiles to the brain parenchyma, so they can be used as a model
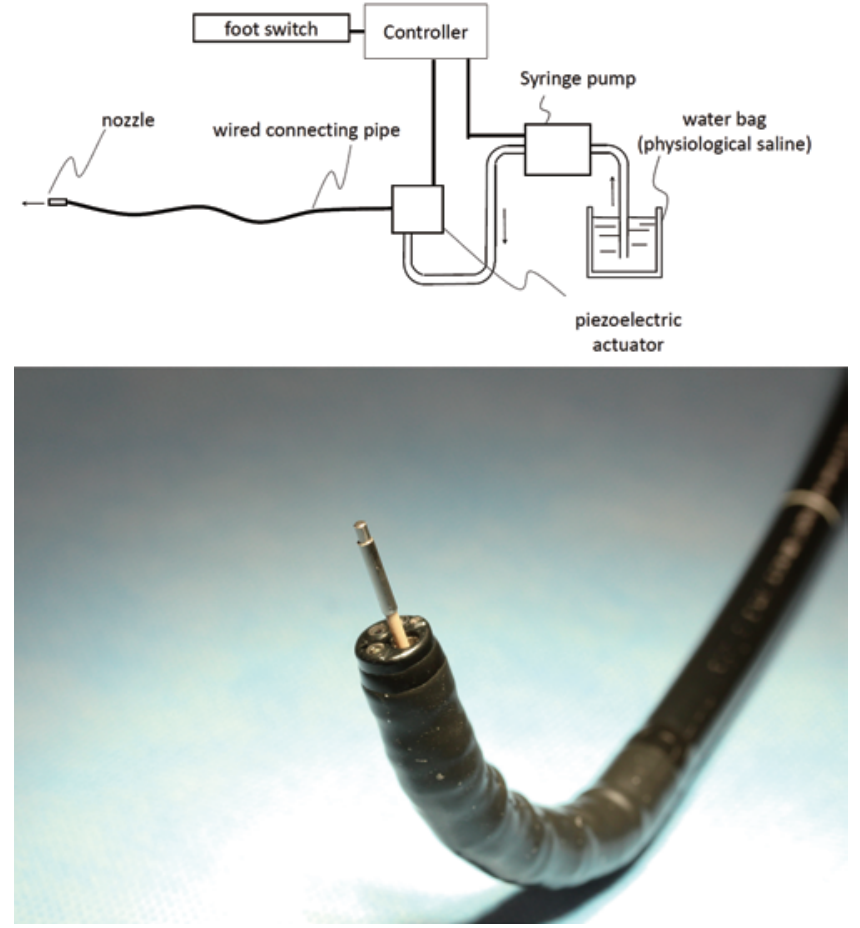

FIG. 1. Upper: Scheme of the piezoelectric ADPJ. Lower: The ADPJ introduced into the flexible endoscope.

of brain tissue..$^{17,21}$ The gelatin specimen was immersed into physiological saline, and the nozzle of the ADPJ was placed perpendicularly to the gelatin surface. A pulsed or continuous-flow water jet was ejected at different flow rates, and the cutting depth was measured.

\section{Evaluation of Safety in Brain Phantom Experiments}

A small cassette representing the ventricle was made of lucent 2-mm-thick acrylic plate. The volume inside was adjusted to $10 \mathrm{ml}$, conforming to the average size of the third ventricle in patients with hydrocephalus. ${ }^{16}$ The cassette was filled with physiological saline and a path for water drainage was made. To monitor the inside conditions, a pressure sensor (MicroSensor; Codman) and a thermometer (NCF600; CHINO Corp.) were used. The water jet device was introduced and a pulsed or continuous water jet was ejected.

\section{Evaluation of Dissection in Porcine Ventricles}

All animal procedures and protocols were approved by the institutional review board of the Center for Laboratory Animal Research of Tohoku University. Ten healthy 3-month-old domestic pigs were subjected to anesthesia induction with medetomidine hydrochloride $(0.06 \mathrm{mg} /$ $\mathrm{kg})$ and midazolam $(0.03 \mathrm{mg} / \mathrm{kg})$. Intravenous access was obtained using a catheter placed in the ear vein, and pentobarbital $(10-20 \mathrm{mg} / \mathrm{kg}$ ) was infused. Subsequently, the animals were intubated with cuffed endotracheal tubes. General anesthesia was maintained with isoflurane (1\%$2 \%$ ) or an intravenous infusion of propofol $(2-5 \mathrm{mg} / \mathrm{kg} / \mathrm{hr}$ ). The animals received continuous ventilation appropriate 
to body weight. During the whole procedure, heart rate, oximetry, arterial blood pressure, and core temperature were monitored and maintained at physiological levels.

First, procedures for other organs such as the liver and esophagus were performed. Subsequently, the animals were killed with an injection of potassium chloride and the whole brain was harvested. The cadaveric brains were cut along the interhemispheric fissure to expose the ventricle wall. To maintain the in vivo condition, the specimens were immediately immersed into physiological saline. The ventricle walls were dissected with the ADPJ, which was introduced into the flexible endoscope (CLV-260, GIF TYPE Q260 J; Olympus). The ADPJ conditions were voltage $30 \mathrm{~V}$, flow rate $5 \mathrm{ml} / \mathrm{min}$, and frequency $200 \mathrm{~Hz}$. For the in vivo experiment, a craniotomy was performed in anesthetized animals. After dural incision the cortex of a small area was microsurgically resected to make the cortico-ventricle window, and then a clear sheath with a diameter of $12 \mathrm{~mm}$ (Neuroport, Olympus) was inserted toward the ventricle. The ventricle walls were dissected with the ADPJ, which was introduced using the flexible endoscope (CLV-260, VEF TYPE V; Olympus). The ADPJ conditions were the same as above.

\section{Histological Examination}

Dissected specimens were immediately fixed with $4 \%$ formalin solution, and embedded into paraffin. The blocks were sliced into $2-\mu \mathrm{m}$-thick sections, stained with $\mathrm{H} \& \mathrm{E}$, and examined under a light microscope.

\section{Results}

\section{Water Jet Pressure and Standoff Distance}

To obtain the effective penetrating strength of the water jet, the relationship between the standoff distance and water pressure of the ADPJ was examined. The water pressure was plotted at different planes (Fig. 2). The water pressure was adequate at approximately 6.5 bar at a distance of $0.5 \mathrm{~mm}$, and was maintained to $1 \mathrm{~mm}$. After that, the pressure rapidly decreased toward $50 \%$ at $2 \mathrm{~mm}$, and to less than $10 \%$ at $3.5 \mathrm{~mm}$.

\section{Dissection Depth in the Phantom Brain Model}

Gelatin specimens were dissected with the pulsed or continuous water jet with different flow rates and the dissection depth was measured (Fig. 3). Based on the findings of the standoff distance above, the distance between the nozzle and gelatin was set at $0.5 \mathrm{~mm}$ to obtain effective penetrating strength. Less water was required for the pulsed water jet compared with the continuous-flow water jet to obtain equal dissection depth. The pulsed water jet required $5 \mathrm{ml} / \mathrm{min}$ to dissect to $2.5-\mathrm{mm}$ depth, whereas the continuous-flow water jet required $15 \mathrm{ml} / \mathrm{min}$.

\section{Safety in the Phantom Ventricle Model}

To confirm the safety of the ADPJ, the pressure and temperature were examined using the device in a closed system representing the intraventricular condition that was filled with physiological saline and open or closed water drainage. With closed drainage, the internal pressure increased with the flow rate. However, with open drainage, the internal pressure did not increase and was controlled at the baseline (Fig. 4). Temperature did not change during the 5-minute trial period (data not shown).

\section{Effective and Safe Dissection of Porcine Ventricle Wall}

Cadaveric porcine brains were immediately immersed into physiological saline (Fig. 5A) and the ventricle wall was dissected using the ADPJ introduced into the flexible endoscope. The dissection line crossed the vessels on the surface of the ventricle wall. The ventricle wall was dis-

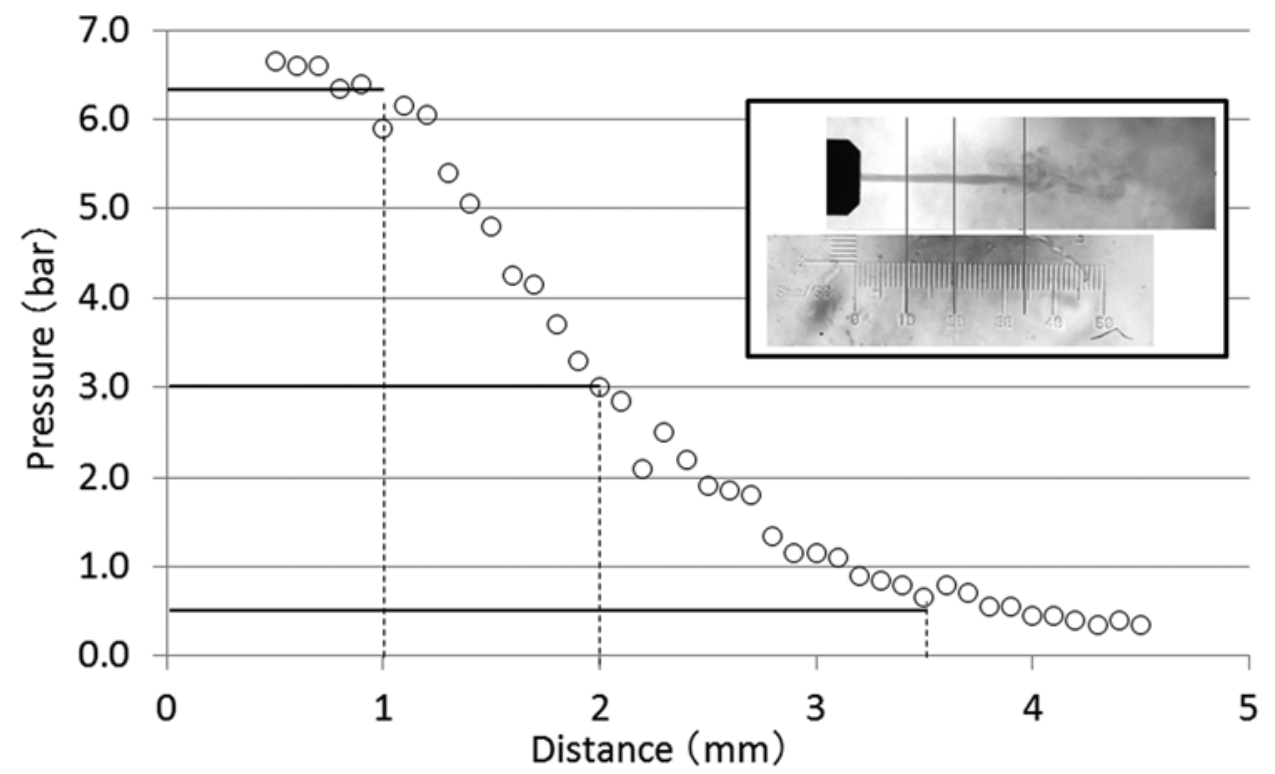

FIG. 2. Graph showing water pressure and standoff distance between the nozzle tip of the ADPJ and the target surface. The inset is a photograph showing an ADPJ ejecting water, combined with a millimeter scale. 


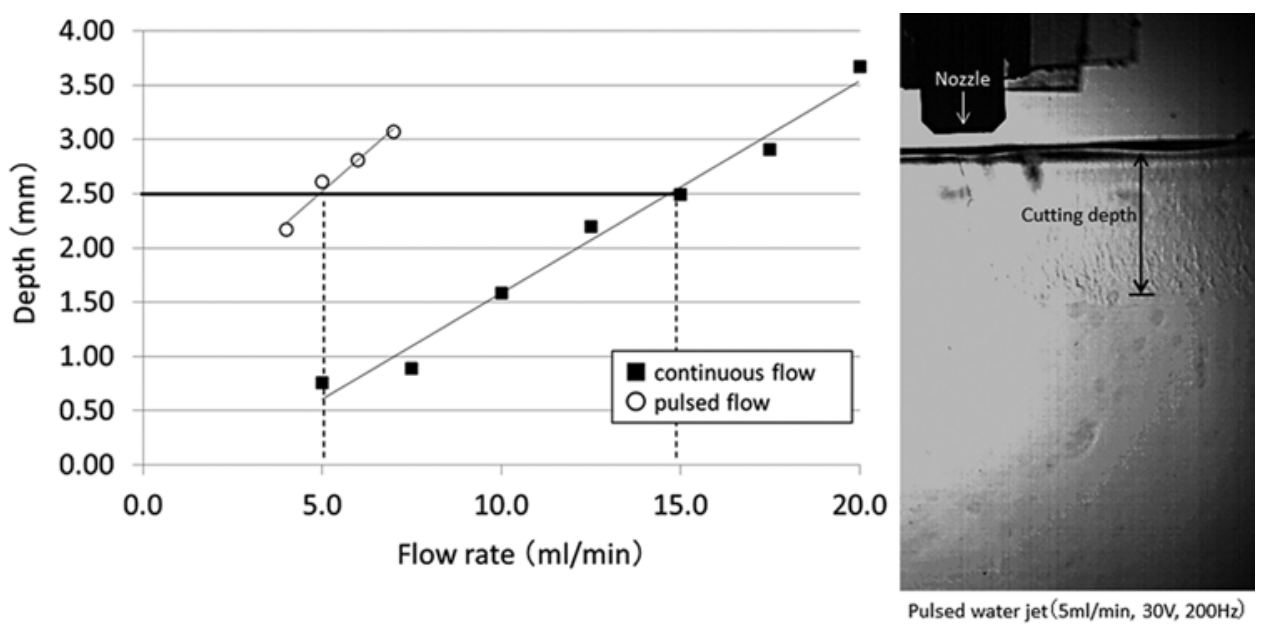

FIG. 3. Left: Graph showing a comparison of the relationship between flow rate and cutting depth using the continuous-flow jet and the pulsed water jet. Right: Photograph showing gelatin penetration with the ADPJ.

sected but these vessels were preserved, as shown in Fig. 5B. Histological examination of the dissected ventricles showed that the ependymal layer and subependymal parenchyma were effectively dissected, whereas large vessels on the ventricle surface were preserved (Fig. 5C), as was the microvasculature of the parenchyma along the dissection plane (Fig. 5D).

To confirm the efficacy and safety of ADPJ in vivo, the flexible endoscope was introduced into the ventricle through the cortico-ventricle window and the ventricle wall was dissected with the ADPJ under continuous irrigation (Fig. 6 left). Histological examination demonstrated the effective dissection of the parenchyma with vessel preservation (Fig. 6 right).

\section{Discussion}

This feasibility study of ADPJ application for flexible neuroendoscopic surgery demonstrated the safety and efficacy of the ADPJ compared with the continuous-flow

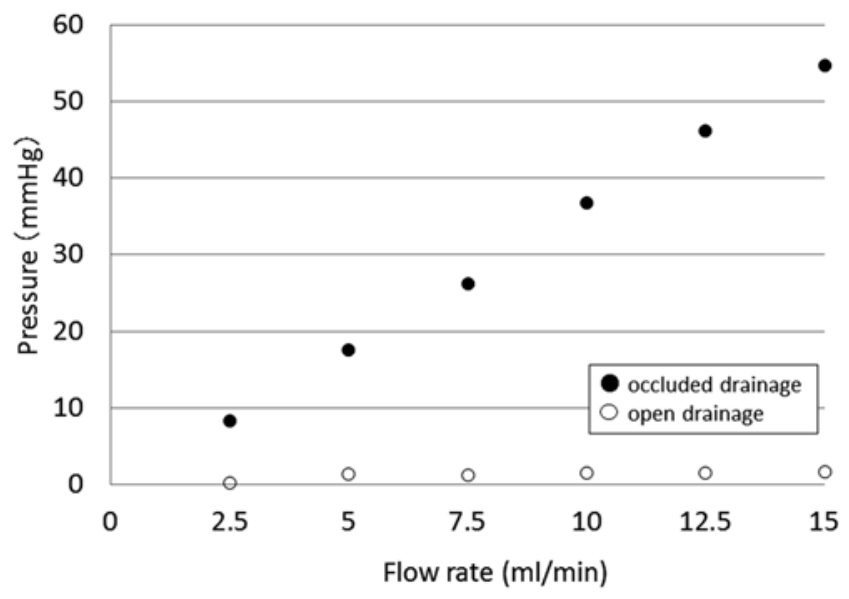

FIG. 4. Plot showing internal pressure of the ventricle model with closed or open drainage. water jet. Therefore, the ADPJ is a promising device that can avoid bleeding when used with the flexible endoscope for the treatment of diseases of the ventricles.

Elevation of ICP is one of the potential risks during flexible neuroendoscopic surgery, ${ }^{2}$ and water injection inside the ventricles can lead to intracranial hypertension. The ADPJ required a smaller amount of water for tissue dissection compared with the continuous-flow water jet. Dissection to a depth of $2.5 \mathrm{~mm}$ was achieved using the ADPJ with $5 \mathrm{ml} / \mathrm{min}$ flow rate, which required one-third the amount of water required by the continuous-flow water jet (Fig. 3). Although ICP elevation can occur if the water drainage is not open (Fig. 4), the present findings indicated that the ADPJ can minimize such risks and avoid severe complications.

Previous studies have shown that the water jet can dissect soft tissue with vascular preservation under dry field conditions. The present study showed that gelatin with elasticity equivalent to brain parenchyma could be dissected effectively even under immersed conditions (Fig. 3 right). Moreover, the ADPJ could also dissect the ventricle wall of the porcine brain ex vivo and in vivo (Figs. 5 and 6 ). We previously reported that the pulsed water jet cannot penetrate membranous structures such as the arachnoid and pia mater, so that the nerves, vessels, and intact parenchyma can be preserved. ${ }^{10,11}$ To dissect lesions beneath the parenchyma, the membrane should be sharply cut before ADPJ application. On the other hand, the ventricle wall is covered with ependymal layer without pia mater. These histological characteristics allow the ADPJ to directly dissect the ventricle wall. The vascular structures were totally preserved even with direct application to the vessels (Fig. 5D). Microvasculature beneath the ependymal layer was also preserved along with the tract. Therefore, the ADPJ can safely dissect the ventricles with vascular preservation.

The present report has several limitations. First, our dissection study with porcine brain might still be preliminary. Cadaveric brains can become hard when exposed to air even if the whole brain is immediately immersed into 


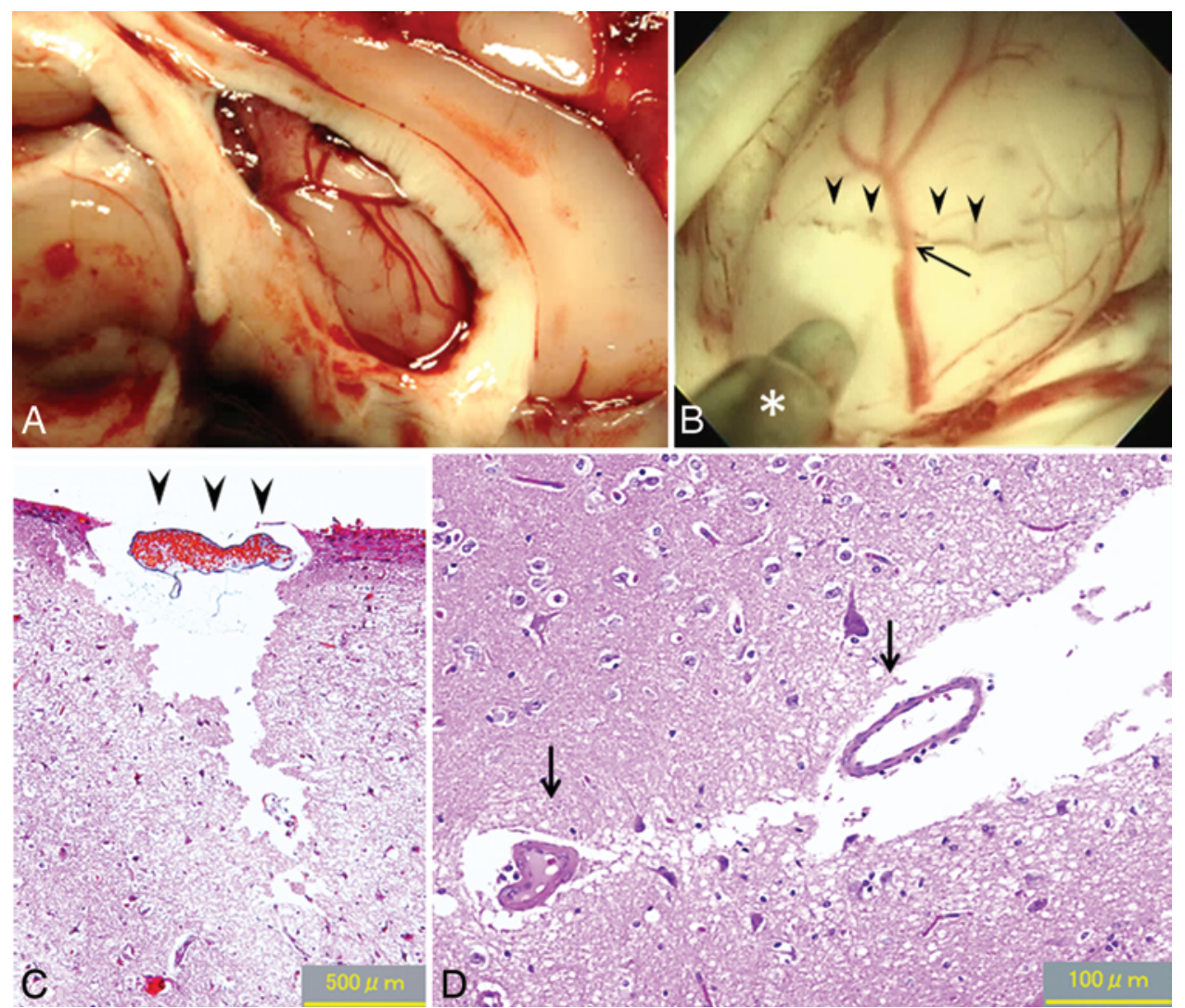

FIG. 5. A: Photograph showing cadaveric porcine brain. B: Endoscopic view showing that the ventricle wall had been dissected (arrowheads) with the ADPJ (asterisk), and that vessels on the ventricle surface (arrow) were preserved. C and D: Photomicrographs of the ventricle wall dissected with the ADPJ. C: The ependymal layer and parenchyma were dissected and the surface vessel was preserved (arrowheads). H \& E, original magnification $\times 40$. D: High-magnification image showing that the subependymal small vessels were also preserved (arrows). $\mathrm{H} \& \mathrm{E}$, original magnification $\times 200$.

physiological saline for application of the ADPJ. We also performed in vivo experiments to overcome this problem. Cortical resection was required to introduce the flexible endoscope, because the ventricle volume is small. Further validation in an in vivo setting, such as an animal model of hydrocephalus, is required. Second, other organs such as the liver and esophagus were used for an in vivo study
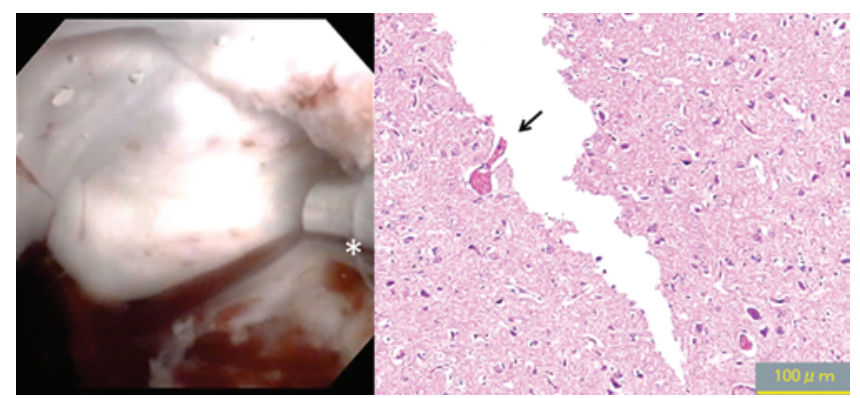

FIG. 6. Left: Ventricle wall of a living pig was dissected with the ADPJ (asterisk). Right: Photomicrograph of the ventricle wall dissected with the ADPJ. High-magnification image showing that the subependymal small vessels were also preserved (arrow). $\mathrm{H}$ \& E, original magnification $\times 200$. before the brain was harvested. This time course of procedures might not be negligible in terms of tissue elasticity changes. However, the animals were still alive even after the procedures, and were killed to obtain the brain for examination.

\section{Conclusions}

The pulsed water jet device was applied using the flexible neuroendoscope. The ADPJ could effectively and safely dissect the ventricle walls with minimal risk of bleeding and ICP elevation. To achieve optimal surgical results under clinical conditions, we would like to emphasize that the ADPJ is a potential dissection device for flexible neuroendoscopic surgery.

\section{References}

1. Baer HU, Metzger A, Barras JP, Mettler D, Wheatley AM, Czerniak A: Laparoscopic liver resection in the Large White pig--a comparison between waterjet dissector and ultrasound dissector. Endosc Surg Allied Technol 2:189-193, 1994

2. Chowdhry SA, Cohen AR: Intraventricular neuroendoscopy: complication avoidance and management. World Neurosurg 79 Suppl:S15.e1-S15.e10, 2013

3. Fletcher DA, Palanker DV: Pulsed liquid microjet for microsurgery. Appl Phys Lett 78:1933-1935, 2001 
4. Hata Y, Sasaki F, Takahashi H, Ohkawa Y, Taguchi K, Une Y, et al: Liver resection in children, using a water-jet. J Pediatr Surg 29:648-650, 1994

5. Izumi R, Yabushita K, Shimizu K, Yagi M, Yamaguchi A, Konishi K, et al: Hepatic resection using a water jet dissector. Surg Today 23:31-35, 1993

6. Jakob S, Kehler U, Reusche E, Friedrich HJ, Arnold H: [Endoscopic use of the water jet dissector in the cerebral ventricle system - an experimental study.] Zentralbl Neurochir 61:14-21, 2000 (Ger)

7. Nakagawa A, Hirano T, Jokura H, Uenohara H, Ohki T, Hashimoto T, et al: Pulsed holmium:yttrium-aluminumgarnet laser-induced liquid jet as a novel dissection device in neuroendoscopic surgery. J Neurosurg 101:145-150, 2004

8. Nakagawa A, Kumabe T, Kanamori M, Saito R, Hirano T, Takayama K, et al: [Clinical application of pulsed laserinduced liquid jet: preliminary report in glioma surgery.] No Shinkei Geka 36:1005-1010, 2008 (Jpn)

9. Nezhat CH, Nezhat F, Seidman DS, Nasserbakht F, Nezhat C, Roemisch M: A new method for laparoscopic access to the space of Retzius during retropubic cystourethropexy. J Urol 155:1916-1918, 1996

10. Ogawa Y, Nakagawa A, Takayama K, Tominaga T: Pulsed laser-induced liquid jet for skull base tumor removal with vascular preservation through the transsphenoidal approach: a clinical investigation. Acta Neurochir (Wien) 153:823 830, 2011

11. Ogawa Y, Nakagawa A, Washio T, Arafune T, Tominaga T: Tissue dissection before direct manipulation to the pathology with pulsed laser-induced liquid jet system in skull base surgery--preservation of fine vessels and maintained optic nerve function. Acta Neurochir (Wien) 155:1879-1886, 2013

12. Papachristou DN, Barters R: Resection of the liver with a water jet. Br J Surg 69:93-94, 1982

13. Penchev RD, Losanoff JE, Kjossev KT: Reconstructive renal surgery using a water jet. J Urol 162:772-774, 1999

14. Persson BG, Jeppsson B, Tranberg KG, Roslund K, Bengmark S: Transection of the liver with a water jet. Surg Gynecol Obstet 168:267-268, 1989

15. Sato C, Nakano T, Nakagawa A, Yamada M, Yamamoto H, Kamei T, et al: Experimental application of pulsed laserinduced water jet for endoscopic submucosal dissection: mechanical investigation and preliminary experiment in swine. Dig Endosc 25:255-263, 2013

16. Schwartz TH, Ho B, Prestigiacomo CJ, Bruce JN, Feldstein NA, Goodman RR: Ventricular volume following third ventriculostomy. J Neurosurg 91:20-25, 1999
17. Seki S, Iwamoto H: Disruptive forces for swine heart, liver, and spleen: their breaking stresses. J Trauma 45:1079-1083, 1998

18. Seto T, Yamamoto H, Takayama K, Nakagawa A, Tominaga T: Characteristics of an actuator-driven pulsed water jet generator to dissecting soft tissue. Rev Sci Instrum 82:055105, 2011

19. Shekarriz B, Shekarriz H, Upadhyay J, Wood DP Jr, Bruch HP: Hydro-jet dissection for laparoscopic nephrectomy: a new technique. Urology 54:964-967, 1999

20. Shekarriz H, Shekarriz B, Kujath P, Eckmann C, Bürk C, Comman A, et al: Hydro-jet-assisted laparoscopic cholecystectomy: a prospective randomized clinical study. Surgery 133:635-640, 2003

21. Yamada M, Nakano T, Sato C, Nakagawa A, Fujishima F, Kawagishi N, et al: The dissection profile and mechanism of tissue-selective dissection of the piezo actuator-driven pulsed water jet as a surgical instrument: laboratory investigation using swine liver. Eur Surg Res 53:61-72, 2014

\section{Disclosure}

This study was funded in part by a grant from the Japanese Foundation for Research and Promotion of Endoscopy. This work was also supported in part by the Translational Research Network Program from the Japanese Ministry of Education, Culture, Sports, Science, and Technology (MEXT), JSPS KAKENHI Grant Number 26462196. Drs. Nakagawa and Tominaga received research support for this study from Seiko Epson Co., Ltd., under a collaborative research contract with Tohoku University, and Dr. Nakagawa is a patent holder with Seiko Epson Co., Ltd.

\section{Author Contributions}

Conception and design: Kawaguchi, Nakagawa. Acquisition of data: Kawaguchi. Analysis and interpretation of data: Kawaguchi. Drafting the article: Kawaguchi. Approved the final version of the manuscript on behalf of all authors: Kawaguchi. Study supervision: Nakagawa, Endo, Fujimura, Sonoda, Tominaga.

\section{Correspondence}

Tomohiro Kawaguchi, Department of Neurosurgery, Tohoku University Graduate School of Medicine, 1-1 Seiryo-machi, Aoba-ku, Sendai, Miyagi 980-8574, Japan.email: kawaguchi@ nsg.med.tohoku.ac.jp. 